\title{
Cosmology between two wars. Einstein's revolution and alternative models of the Universe
}

\author{
Anna Curir \\ INAF-Osservatorio Astronomico di Torino, \\ Strada Osservatorio 20, 10025 Pino Torinese, Italy \\ email: curir@oato.inaf.it
}

\begin{abstract}
The personality of Edward Milne is examined, deepening the historical period in which his Cosmological theory was produced. Is suggested that Milne's kinematical Cosmology can be regarded as a kind of 'resistance' to the scientific revolution deriving from the new Einstein's theory of gravitation. Is investigated the deep philosophical meaning of the metrics on curved manifolds as far as the representation of the universe is concerned, and the importance of the cosmological theories in the epistemology and in the evolution of science is stressed.
\end{abstract}

Keywords. General relativity, cosmology, scientific revolutions, Milne

\section{Paradigms and Scientific Revolutions}

According to Kuhn (1962), a scientific revolution appears at the end of a long period of so-called normal science inside a well established paradigm, namely a general framework containing the fundamental laws governing a natural phenomenology (e.g. the Newtonian paradigm of gravitation). It is announced by anomalies that cannot be explained by the old paradigm.

A scientific revolution is a big crisis period, painful for the scientists who lose the references where they have been working (Curir 2005). A 'resistance' can be developed against the affirmation of the new paradigm. There are scientists who abandon their research activity because they are unable to cope with the lack of certainties they were used to work with. But in the end the new paradigm is asserted. Within the new paradigm old facts are seen with new eyes and interpreted through new laws. For a definitive affirmation of the new paradigm, it is important that the 'anomalies' of the old paradigm can be explained with new one. Therefore one would think that the Einstein revolution produced by General Relativity lasted from 1915 (the year of the publication) to 1919 (when the Royal Astronomical Society expedition to measure the light deflection due to the sun, produced the first experimental proof of the new theory). But in cosmology the fight between the old paradigm and the new one dragged on.

I propose to explain this peculiar evolution of the alternative Cosmological Models with the big social crisis in the decades when these theories were developed. Such a general crisis enhanced the fears and uncertainties linked to the abandon of the old scientific paradigm. And the resistance to the new theories was even stronger, orienting some scientists toward theistic principles.

\section{The social and economical crisis between the wars}

The Great Depression was a worldwide economic downturn starting in most places in 1929 and ending in the 1930s or early 1940s for different countries. It was the largest 
economic depression in the 20th century. The Great Depression originated in the United States; the starting date is set at the stock market crash on October 29, 1929. The effects of the Depression were profound throughout Europe, though the greatest impact was on Germany, Austria, England and Poland.

In the recent months many economists made parallelism between the actual Mondial economical crisis and the Great Depression, therefore it can be particularly interesting today to focus on the influence that the economical crisis between the two wars had on culture and science. Historians like Spengler and Arnold Toynbee and economists like Keynes considered these years as a beginning of the decline of humanity. Toynbee called 1931 annus terribilis and compared the situation to the one of III century, when we had the fall of the classical civilisation. Toynbee conceived a possible overcome of such a situation with the construction of a new, less materialistic society.

Even before the beginning of the Big Depression, poets like Yeats, Eliot (The waste land) and Kraus ( The last days of mankind) suggested catastrophic scenarios of the future for humanity. In his pamphlet Mass civilisation and Minority Culture Leavis (1930) made a very weak estimation of the prospects for resistance to the forces of cultural destruction, and his pessimistic reading of history produces a negative perspective for the coming years. In a later book written with Thompson (1933) Culture and environment, speaking about "the death of Old England and the replacement of the more primitive nation by a modern state" he comments: "The Old England was the England of the Organic Community and in what sense it was more primitive than the England that has replaced it needs pondering".

It was inevitable that science itself, namely one of the forces that helped the developing of the industrial society, could somehow be accused of being responsible of the big crisis. A strong debate was on in important scientific journals: I quote here a paper on Nature, with the title Unemployment and hope by Linn Cass (1930). In this article it is said that the new capitalism, based on individual profit and wild industrialism and on the exploitation of natural resources is responsible for the crisis. In the same paper on can see the suggestion of a return to old times, a refusal of modern structures of production (again, nowadays we have similar reactions...).

In the journal Scientia (1932) the French mathematician Borel felt the need of writing a paper with the title: La science est-elle responsable de la crise mondiale? where he defends the scientific progress which is accused to be the basis of the new industrial progress, from where the big crisis originated.

\section{Alternative Cosmologies and their different approaches}

As Kragh (1996) pointed out, there is a problematical relationship between the historian's kind of history and the kind of history written by scientists. One aspect of the scientists' history is the temptation to write the history of science so as it fits with the most modern views of physics and ignore the false trails that may seem irrelevant. Cosmology in particular is full of false trails and even today one cannot be confident that the dominant Dark Matter-Energy paradigm is the final answer. So the development of this field in particular should be treated with historical sensitivity. One example of an distorted historical perspective is the poor attention for Edward Milne's research of the 1930s . Indeed, most astronomers ask why it should be recalled when it turned out to be a failure. From an historical point of view Milne was a key person in modern cosmology during the critical phase between the 1930s and the 1940s.

In the period between the two wars we have the following alternative cosmologies: I) the Relativistic Cosmologies, that are solutions of the Einstein's field equations applied to the whole Universe (the first solution date of 1917). 
II) Milne's deductive Cosmology, proposed in 1933: a kinematical expanding Universe, no gravity, Minkowsky's space-time (Milne 1933, 1935).

III) In 1948 is proposed the Steady State Cosmology: a Universe homogeneous in space and in time, but accounting for expansion (continuous creation of matter). Even if this model was proposed after the second world war, I include it here as an 'extremely late resistance' to the Relativistic Cosmology.

In Relativistic Cosmologies the Universe has no outside. The Milne Universe does have an outside, the whole Universe of galaxies get created at a single point in flat spacetime (Milne tried to justify this on theological grounds), and thereafter occupies the interior of a bubble that expands into previously empty space. The galaxies are treated as non-gravitating test particles. They all shoot out at different speeds inside the bubble. The closer they are to the speed of light, the nearer they will be to the surface of the bubble. Milne's Cosmology can be assimilated to the zero-density limit of the expanding Friedmann-Robertson-Walker (FRW) metrics with no cosmological constant. The steady state Universe has no outside, no curvature, is infinite and in expansion, but homogeneous in space and time.

Cosmology is conditioned by principles or assumptions that are un-verifiable. The most important of these assumptions is the Cosmological Principle which consists of the postulates of spatial homogeneity and isotropy. These properties belong to Milne's deductive Cosmology and to Relativistic Cosmologies. One should anyway credit the Steady State Cosmological theory (Bondi, Gold and Hoyle) the merit of relativising also our idea of time (with the Perfect Cosmological Principle).

The major difficulty in the production of a Cosmology from General Relativity was the link between the small scale and the Universe scale: while Euclide works at small scale you need Riemann at larger scale. If physics is only understood locally, the Universe becomes at best an educated guess: Hermann Weyl was worried about a wild pluralism that could be developed on geometrical basis.

Einstein was following his philosophy proposing to eliminate any physical property linked to the absolute nature of space or time. Deeply influenced by Mach, he put the inertia as linked with the masses and not with the empty space. And the link between geometry and density of General Relativity suggested the possibility of solving in an unique way the Cosmological problem: that became the initial Einstein's program. The idea was to keep the average density constant and to link the radius of the Universe to the density (in the hypothesis of a static Universe). Following this program Einstein produced the first cosmological solution of his equations, and presented it in a report of the Prussian Academy of Sciences (Einstein 1917).

The cosmic expansion of the Universe had not been discovered at the time, so he proceeded to build a static Universe by adding to the field equations, a cosmological constant $\Lambda$. This seemed to solve a problem that troubled even Newton: why did the entire Universe not collapse under its own gravitational attraction. The $\Lambda$ introduces a screening length allowing the motion of stars and nebulae to approach equilibrium. The gravitational field equations balance, in this way, but this model Universe is unstable to expansion or collapse. This first attempt, however, revealed to be unsatisfactory, since De Sitter proposed a new solution with $\Lambda$ describing a finite and empty Universe. Einstein believed that his theory satisfied Mach's principle: the geometrical structure of spacetime is fully determined by its matter content. De Sitter, with his empty solution showed that this is not true.

With the contribution of the Russian mathematiscian Friedmann (1922) one started to realize that by admitting the non-staticity of the Universe, it was possible to get rid of 
the cosmological constant. It was indeed formulated the first solutions for an expanding Universe (Friedmann 1924)

Cosmology is different from other domains of physics. The physics normally deal with objects or events which can be generalized or repeated; but there is only one Universe and the Big Bang is a non-repeatable event.

The peculiar unique subject of Cosmology moved scientists like Milne to search for a unique model following a few axioms in a deductive way. Such a deductive method had a lot of success in that period and was adopted also by Robertson and Bondi (one of the fathers of Steady State Universe). Milne wanted to give an answer to a void of authority in the field of the Cosmology. An answer to a situation where the Einstein's program (to deduce Cosmology from the density of the Universe in an unique way) failed, leaving space for many possibilities: Universe finite or infinite, static, expanding (the solutions produced by De Sitter, Friedmann, Lemaître, Eddington...).

Milne himself said that his theory was a kind of answer to the discredit on which General Relativity has thrown the Cosmology to the eyes of physicists. His kinematical Cosmology represented a return to classical sources (Leibniz, Newton), a return to a teistic vision of the Universe. Its Cosmological Principle is indeed based on the faith in the rationality of the Creator.

In this difficult historical period between two wars, with a big economical crisis we have two approaches to the Cosmology which both can explain the phenomenological observations: the red shift of galaxies (Slipher 1913) and its relation with their distances (Hubble, 1929). Relativistic Cosmologies are based on extrapolation on large scale structure of field equations based on local scale. Milne's theory on the contrary is deductive: all is derived from the Cosmological principle.

Einstein's Cosmology was the real revolution: it extrapolated to Universe scale the new local physics. Milne's Cosmology was the return to the old principles, to Newton and to God: the Universe deducted from a single divine principle. For these reasons Milne's theory could be regarded as a cultural answer: it offers old certainties in a period of severe economical crisis. But his theory could also be regarded as an example of 'resistance' to a scientific revolution

On the other hand, Michele Besso (1917) was saying that Einstein's General Relativity is a theory where the speculative thinking (die Spekulation) is superior to the empirical elements (der Empirie). Einstein (1918) replied to this with the sentence: to be acceptable, a theory must be built on facts which can be generalized. He also said that "we can't consider Riemann's results as pure speculations; The Gauss's contribution is that of having formulated the laws of deplacements of small rigid bars (the ds of the metric) on a surface. Without such idea of small bars all the reasoning would have been impossible". These words have the aim of overcoming the dichotomy between the conceptual and the empirical, between mathematics and physics. Einstein declared his according with Kant's sentence "The real is not given to us but put to us (aufgeben) (by way of a riddle)", but he stressed the disagreement with him in the sense that the categories are not immutable but free convention in a logical sense.

Einstein's revolution was also this new way of regarding the cathegories as 'conventions' and a new way of regarding the facts to be generalised: the small riemaniann bars are like empirical elements, but they allow the extrapolation of the metric to curved spacetimes and to very large scale! We must keep the memory of the terrestrial origin of the little $d s$ bar. Hermann Weyl calls Riemann's theory the geodesy of the multidimensional geometry. 


\section{Role of Milne's Cosmology in this period and its legacy}

According to Popper (1934) the vulnerability of theories is a positive feature: their refutation represents knowledge and makes it easier to find a better theory. Being refuted, Milne's Cosmology and the Steady State model played an important role in the progress of Cosmology.

Before Milne, there were many cosmological models under consideration, on the basis of General Relativity. But Milne's emphasis on the Cosmological Principle, made more explicit a property that most of these models already had. The gravityless nature of his cosmology also made much clearer what you really needed gravity for, and what you didn't need it for. Finally, the Milne's model is today a very good pedagogical tool.

\section{The impact of Cosmology on modern science}

The doubt that astrophysics is not a proper science goes back in time and was expressed by Auguste Comte in his Cours de Philosophie Positive in the 1830s, when he claimed that we could never have positive knowledge about the sun or the stars. His idea of a secure knowledge was limited to those objects that can be reproduced or interfered with in laboratory experiments. As I said before one cannot experiment with the whole Universe, but this is not an unusual situation in science: it is shared by all those sciences which deal with the past, like for example paleontology and geology.

On the other hand modern Astronomy and Cosmology are an important example of a progressive abandon of a science based on human centrality in favour of a perspective where the man is projected in a neutral and objective space. Astronomy and Cosmology, stressing for example the fact that the optical perception of star luminosity does not give account of all the field of existence of stars and galaxies, show that the construction of scientific theories is connected with the refuse of the limit of the simple empirical reality. As Thom \& Noël (1993) explained "every theory consists in the refusal of the simple observed reality, in the construction of a bigger virtual reality containing the observed one and in the description of such constraints governing the propagation of the real in the virtual'.

Moreover Astronomy and Cosmology put us in a continuous contact with a reality which is deeply "historical and evolutive". The observed phenomena and the reality of Cosmology are deeply characterised by a time stream. An atomical physicist can ignore the history of the reality of which he is studying the model, but a cosmologist must always take into account the history and the evolution of the phenomena he is dealing with.

This suggestion is very important since it forces the scientist to take into account the role of the historical dimension in the natural sciences, not only in the evolution of ideas or of populations; that means, to think in terms of a natural history which links somehow Cosmology, Biology and historical sciences. Cosmology, with such a structural opening suggests a conception of the man as floating in an "open time stream" so that for such a man it is easier a critical position against a petrified outlook on nature (Engels, 1956).

\section{References}

Besso, M. 1917, in Correspondance Einstein-Besso 1903-1955 (1972 translation) (Paris: Hermann)

Borel, E. 1932, Scientia 51, 99

Curir, A. 2005, Giornale storico del Centro Studi di Psicologia e Letteratura, 1, 113

Einstein, A. 1917, Preussische Akademie der Wissenschaften, Sitzungsberichte, 142 
A. Curir

Einstein, A. 1918, in Correspondance Einstein-Besso 1903-1955 (1972 translation) (Paris: Hermann)

Engels, F. 1956, Dialettica della Natura (Roma: Editori Riuniti)

Friedmann, A. 1924, Z. fur Phys. 21, 326

Hubble, E. 1929 Proceedings of the National Academy of Sciences 15, 168

Kuhn, T. 1962, The structure of scientific revolutions (Chicago: University of Chicago Press)

Kragh, H. 1996, Atti del XVI Convegno Nazionale di Storia della Fisica e Astronomia (Como:Centro Volta) http://www.brera.unimi.it/sisfa/atti/1996/kragh.html

Leavis, F. R. 1930, Mass Civilization and Minority Culture (Cambridge: The Minority Press)

Lin Cass, W. G. 1930 Nature 125, 345

Leavis, F. R., Thompson, D. 1933, Culture and environment (London:Chatto and Windus)

Milne, E. A. 1933, Z. Astrophys. 6, 1

Milne, E., A. 1935, Relativity, Gravitation and World-Structure (Oxford: Clarendon Press)

Popper, K. 1934, The logic of scientific discovery (London: Hutchinson)

Slipher, V. M. 1913, Lowell Observatory Bulletin, 1, 56

Thom, R. \& Noël, E. 1993, Predire n'est pas expliquer (Paris: Flammarion) 